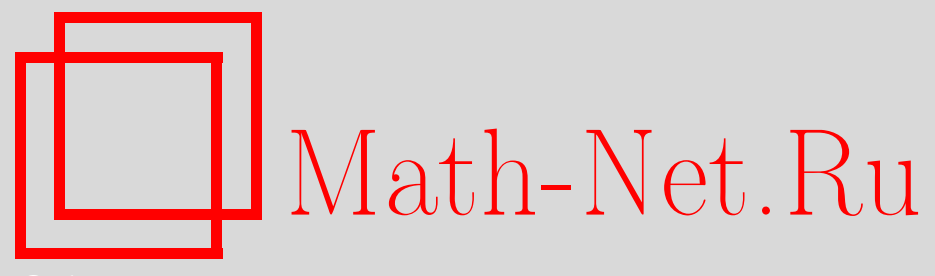

В. А. Копытцев, В. Г. Михайлов, Явные оценки точности пуассоновской аппроксимации для распределения числа решений случайных включений, Матем. вопр. криптогр., 2015, том 6, выпуск 1, 57-79

DOI: https://doi.org/10.4213/mvk151

Использование Общероссийского математического портала Math-Net.Ru подразумевает, что вы прочитали и согласны с пользовательским соглашением http://www . mathnet.ru/rus/agreement

Параметры загрузки:

IP: 18.207 .199 .55

26 апреля 2023 г., $11: 54: 49$

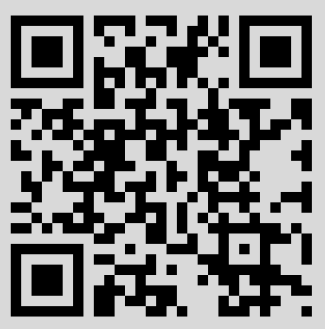




\title{
Явные оценки точности пуассоновской аппроксимации для распределения числа решений случайных включений
}

\author{
В. А. Копытцев ${ }^{1}$, В. Г. Михайлов ${ }^{2}$ \\ ${ }^{1}$ Академия криптографии Российской Федерации, Москва \\ ${ }^{2}$ Математический институт им. В. А. Стеклова РАН, Москва
}

Получено 22.IV.2014

Исследуется точность пуассоновской аппроксимации для распределения числа решений системы случайных включений, принадлежащих заданному множеству попарно не подобных векторов над конечным полем. Приведены численные примеры.

Ключевые слова: системы случайных включений, системы полиномиальных уравнений, пуассоновская аппроксимация

Explicit accuracy estimates for the Poisson approximation to the distribution of the number of solutions of random inclusions

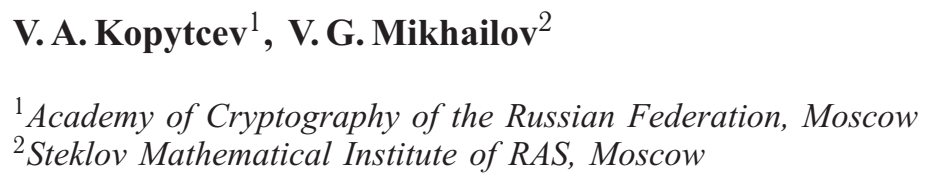

Abstract. We study the accuracy of the Poisson approximation for the distribution of the number of solutions of the system of random inclusions belonging to a given set of pairwise nonproportional vectors over the finite field. Numerical examples are given.

Key words: systems of random inclusions, systems of polynomial equations, Poisson's approximation

Citation: Mathematical Aspects of Cryptography, 2015, vol. 6, no. 1, pp. 57-79 (Russian). 


\section{1. Введение}

Пусть $\xi(D, A, S, B)$ - число решений системы включений

$$
x \in D, \quad A x+S(x) \in B
$$

где $A-$ случайная матрица из элементов поля $K=G F(q)$ размера $T \times n$, $D \subseteq K^{n}$ и $B \subset K^{T}-$ некоторые множества $n$-мерных и $T$-мерных векторов над этим полем, $S(x)-$ произвольное отображение $K^{n}$ в $K^{T}$.

Исследованию условий сходимости при $n, T \rightarrow \infty$ распределения случайной величины $\xi(D, A, S, B)$ к распределениям пуассоновского типа были посвящены работы [1-4]. Как и в этих работах, будем предполагать, что элементы случайной матрицы $A=\left(a_{i, j}\right)$ независимы в совокупности и имеют распределения

$$
\mathbf{P}\left\{a_{i, j}=k\right\}=\frac{1+\Delta_{i, j}(k)}{q}, \quad k \in K, \quad \sum_{k \in K} \Delta_{i, j}(k)=0, \quad i, j \geq 1,
$$

где

$$
\Delta=\max _{i, j, k}\left|\Delta_{i, j}(k)\right|<1 .
$$

Обозначим через $N\left(k_{1}, k_{2}, k_{3}, c, D\right)$ число решений уравнения $k_{1} u^{1}+$ $+k_{2} u^{2}+k_{3} u^{3}=c$ относительно тройки векторов $\left(u^{1}, u^{2}, u^{3}\right) \in D^{3}$, где $k_{1}, k_{2}, k_{3} \in K \backslash\{0\}, c \in K^{n}$. Пусть $N(D)=\max _{k_{1}, k_{2}, k_{3}, c} N\left(k_{1}, k_{2}, k_{3}, c, D\right)$,

$$
\rho(D)=N(D) /|D|^{2} .
$$

Так как уравнение $k_{1} u^{1}+k_{2} u^{2}+k_{3} u^{3}=c$, где $c \in D$ и $k_{1}=k_{2}=1, k_{3}=-1$, имеет решения вида $\left(u^{1}, u^{2}, u^{3}\right)=(c, u, u)$ при всех $u \in D$, то

$$
|D|^{-1} \leq \rho(D) \leq 1 .
$$

Аналогично определим величину $\rho(B)$.

Элементы $x$ и $x^{\prime}$ множества $D$ назовем подобными, если $x^{\prime}=k x$ при некотором $k \in K \backslash\{0,1\}$. Отметим, что в случае $K=G F(2)$ подобные векторы отсутствуют.

В работе [4] была доказана следующая теорема.

Теорема 1. Пусть $K=G F(q)$, множество $D$ не содержит подобных векторов, отображение $S(x)$ не зависит от матрииы $A$, распределение элементов матриць А удовлетворяет условию (2), $0^{n} \notin D$, и при $n, T \rightarrow \infty$ выполнены соотношения $|D| \rightarrow \infty$,

$$
q^{-T}|D||B| \rightarrow \lambda \in(0, \infty), \quad T \Delta \rightarrow 0, \quad \rho(D) \rho(B) \rightarrow 0 .
$$


Тогда

$$
\lim _{n, T \rightarrow \infty}\left|\mathbf{P}\{\xi(D, A, S, B)=r\}-\lambda^{r} e^{-\lambda} / r !\right| \rightarrow 0, \quad r=0,1, \ldots,
$$

причем эта сходимость равномерна относительно отображений $S(x)$.

В настоящей работе оценивается точность пуассоновской аппроксимации в предельной теореме 1. В предположении, что выполнены «неасимптотические» условия этой теоремы и $\Delta=0$, ниже в теореме 2 даны оценки сверху для величин

$$
\left|\mathbf{P}\{\xi(D, A, S, B)=r\}-\tilde{\lambda}^{r} e^{-\tilde{\lambda}} / r !\right|, \quad r=0,1,2, \ldots,
$$

где $\tilde{\lambda}=q^{-T}|D||B|$.

Остальной материал статьи связан с приложениями теоремы 2 к системе включений вида

$$
x \in K^{n} \backslash\left\{0^{n}\right\} \quad A_{1} x+A_{2} f(x)+S(x) \in B,
$$

где $A_{1}$ и $A_{2}$ - случайные матрицы над полем $K$ размеров $T \times n$ и $T \times m$ соответственно, $f(x)=\left(f_{1}(x), \ldots, f_{m}(x)\right): K^{n} \rightarrow K^{m}$ - заданное отображение. Класс включений (5) представляет особый интерес, поскольку содержит системы полиномиальных уравнений

$$
\sum_{\substack{d_{1}, \ldots, d_{n} \in\{0, \ldots, q-1\} \\ 1 \leq d_{1}+\ldots+d_{n} \leq g_{t}}} a_{d_{1}, \ldots, d_{n}}^{(t)} x_{1}^{d_{1}} \ldots x_{n}^{d_{n}}=b_{t}, \quad t=1, \ldots, T,
$$

где $a_{d_{1}, \ldots, d_{n}}^{(t)}-$ независимые в совокупности случайные величины, a $g_{t}$ - целые числа в пределах $1 \leq g_{t} \leq n(q-1)$.

Изучение свойств системы (5) использует следующий прием. Положим $H=K^{n} \backslash\left\{0^{n}\right\}, H_{f}=\{(x, f(x)): x \in H\}$. Число решений системы (5) равно числу решений системы включений вида (1.1):

$$
y \in H_{f}, \quad A y+S^{\prime}(y) \in B
$$

где $y=(x, f(x)), A=\left(A_{1}, A_{2}\right), S^{\prime}(y)=S(x)$. Поэтому для исследования точности пуассоновской аппроксимации распределения числа решений системы (5) можно использовать теорему 2, применяя ее к числу решений $\xi\left(H_{f}, A, S^{\prime}, B\right)$ системы (6). При этом оценку величины $\rho\left(H_{f}\right)$ можно свести к оценке для $\rho\left(\left(K^{n}\right)_{f}\right)$, где

$$
\left(K^{n}\right)_{f}=\left\{(x, f(x)): x \in K^{n}\right\}
$$


так как

$$
\rho\left(H_{f}\right) \leq\left(\frac{q^{n}}{q^{n}-1}\right)^{2} \rho\left(\left(K^{n}\right)_{f}\right) .
$$

В работе приведены оценки сверху и явные выражения для $\rho\left(\left(K^{n}\right)_{f}\right)$ и численные примеры, в которых рассмотрены приложения теоремы 2.

\section{2. Формулировки результатов}

Пусть $\tilde{\lambda}=q^{-T}|D||B|$.

Теорема 2. Пусть $K=G F(q)$, множество $D$ не содержит подобных векторов, отображение $S(x)$ не зависит от матрицы $A$, распределение элементов матриць А удовлетворяет условию (2), где $\Delta=0,0^{n} \notin D$. Тогда при любых неотрицательных изельх числах $r$ и $N$, удовлетворяющих неравенству $N>r+\tilde{\lambda}$, справедлива оценка

$$
\begin{gathered}
\left|\mathbf{P}\{\xi(D, A, S, B)=r\}-\frac{\tilde{\lambda}^{r}}{r !} e^{-\tilde{\lambda}}\right| \leq \frac{2 \tilde{\lambda}^{N+1}}{r !(N+1-r) !} \\
+S_{1, N}(r)+S_{2, N}(r)+S_{3, N}(r),
\end{gathered}
$$

где

$$
\begin{gathered}
S_{1, N}(r)=\rho(D) \sum_{k=r}^{N} \frac{C_{k}^{2} \tilde{\lambda}^{k}}{r !(k-r) !}\left[\left(q+|D|^{-1}\right)^{k-2}-q^{k-2}\right] \\
S_{2, N}(r)=\frac{1}{q^{T}} \sum_{k=r}^{N} \frac{k \tilde{\lambda}^{k-1}}{r !(k-r) !} \\
S_{3, N}(r)=\rho(D) \rho(B) \sum_{k=r}^{N+1} \sum_{j=2}^{k-1} \frac{\tilde{\lambda}^{j}}{r !(k-r) !} .
\end{gathered}
$$

Замечание 1. Из вида оценки (9) следует, что при заданном $r$ и ограниченном $\tilde{\lambda}<C$ малое значение первого члена в правой части (9) достигается за счет выбора значения $N$, а малые значения величин $S_{1, N}(r), S_{2, N}(r), S_{3, N}(r)$ при выбранном $N$ - за счет выбора малых значений $\rho(D),|D|^{-1}$ (как функций параметра $n), q^{-T}$ и величины $\rho(D) \rho(B)$ (как функции параметров $n$ и $T$ ) соответственно.

Введем предельные значения

$$
S_{i}(r)=\lim _{N \rightarrow \infty} S_{i, N}(r), \quad i=1,2,3
$$


выражений (10)-(12) при $N \rightarrow \infty$ (ряды, соответствующие суммам в (10)(12), сходятся).

Первый член в правой части (9) стремится к нулю при $N \rightarrow \infty$, что позволяет получить следующую оценку. Положим,

$$
\varphi_{r}(z)=e^{\tilde{\lambda} z}\left(\tilde{\lambda}^{r+2} z^{r}+2 r \tilde{\lambda}^{r+1} z^{r-1}+\left(r^{2}-r\right) \tilde{\lambda}^{r} z^{r-2}\right) .
$$

Следствие 1. Пусть выполнены условия теоремы 2. Тогда для любого $r=0,1,2, \ldots$

$$
\left|\mathbf{P}\{\xi(D, A, S, B)=r\}-\frac{\tilde{\lambda}^{r}}{r !} e^{-\tilde{\lambda}}\right| \leq S_{1}(r)+S_{2}(r)+S_{3}(r),
$$

zวe

$$
\begin{gathered}
S_{1}(r)=\frac{\rho(D)}{r !}\left[\varphi_{r}\left(q+|D|^{-1}\right)-\varphi_{r}(q)\right], \\
S_{2}(r)=\frac{1}{q^{T}} \frac{\tilde{\lambda}^{r-1}}{r !}(\tilde{\lambda}+r) e^{\tilde{\lambda}},
\end{gathered}
$$

а величины $S_{3}(r), r=0,1,2, \ldots$, вычисляются по формулам (с доопределением по непрерывности при $\tilde{\lambda}=1$ )

$$
\begin{gathered}
S_{3}(0)=\rho(D) \rho(B) \frac{1}{\tilde{\lambda}-1}\left[\left(e^{\tilde{\lambda}}-1-\tilde{\lambda}-\frac{\tilde{\lambda}^{2}}{2}\right)-\tilde{\lambda}^{2}\left(e-\frac{5}{2}\right)\right], \\
S_{3}(1)=\rho(D) \rho(B) \frac{1}{\tilde{\lambda}-1}\left[\tilde{\lambda}\left(e^{\tilde{\lambda}}-1-\tilde{\lambda}\right)-\tilde{\lambda}^{2}(e-2)\right] \\
S_{3}(r)=\rho(D) \rho(B) \frac{1}{r !(\tilde{\lambda}-1)}\left[\tilde{\lambda}^{r} e^{\tilde{\lambda}}-\tilde{\lambda}^{2} e\right], \quad r \geq 2 .
\end{gathered}
$$

Для проведения расчетов по формулам (9)-(12) или (15)-(20) необходимо иметь оценку величины $\rho(D)$. Приведем некоторые примеры для величин $\rho\left(\left(K^{n}\right)_{f}\right)$ (см. (6)-(8)). Рассмотрим отображение $f(x)=\left(f_{1}(x), \ldots, f_{m}(x)\right)$, в качестве части координатных функций $f_{1}(x), \ldots, f_{m}(x)$ которого выступают все произведения вида $x_{1}^{d_{1}} \ldots x_{n}^{d_{n}}$, где $2 \leq d_{1}+\ldots+d_{n} \leq d$, a $d=d(n) \leq(q-$ $-1) n-$ заданное число. В [2] при доказательстве теоремы 5 было показано, что в этом случае

$$
\rho\left(\left(K^{n}\right)_{f}\right) \leq\left(1-q^{-4}\right)^{[n / 2]}
$$


Этот результат был усилен в [3] (неравенство (3.10)) следующим образом. Введем при $l=2,3, \ldots$ множества функций

$$
Q\left(d^{1}, \ldots, d^{l}\right)=\bigcup_{s=1}^{l}\left\{x_{1}^{d_{1}^{s}} \ldots x_{n}^{d_{n}^{s}}\right\}
$$

где $d^{s}=\left(d_{1}^{s}, \ldots, d_{n}^{s}\right) \in\{0,1\}^{n}, s=1, \ldots, l$, и при этом

$$
\begin{gathered}
\left\|d^{s}\right\| \geq 2, \quad s=1, \ldots, l \\
\left\|\left(d_{1}^{s_{1}} d_{1}^{S_{2}}, \ldots, d_{n}^{S_{1}} d_{n}^{S_{2}}\right)\right\|=0, \quad 1 \leq s_{1}<s_{2} \leq l .
\end{gathered}
$$

Согласно условию (23) множества существенных переменных функций $x_{1}^{d_{1}^{s}} \ldots x_{n}^{d_{n}^{s}}$ из набора $Q\left(d^{1}, \ldots, d^{l}\right)$ попарно не пересекаются, а по условию (22) степень нелинейности функций $x_{1}^{d_{1}^{s}} \ldots x_{n}^{d_{n}^{s}}$ больше единицы.

Теорема 3. Пусть $K=G F(q),\left\{f_{1}(x), \ldots, f_{m}(x)\right\} \supseteq Q\left(d^{1}, \ldots, d^{l}\right), l \leq m$. Тогда

$$
\rho\left(\left(K^{n}\right)_{f}\right) \leq \prod_{s=1}^{l}\left(1-q^{-2\left\|d^{s}\right\|}\right) .
$$

В настоящей работе будет показано, что в случае, когда $K-$ простое поле, условия теоремы 3 можно ослабить. Рассмотрим некоторое множество $Q_{n, l}$ многочленов $\left\{\varphi_{1}\left(x_{1}, \ldots, x_{n}\right), \ldots, \varphi_{l}\left(x_{1}, \ldots, x_{n}\right)\right\}$, удовлетворяющих условиям

$$
\begin{gathered}
\operatorname{deg} \varphi_{s}\left(x_{1}, \ldots, x_{n}\right) \geq 2, \quad s=1, \ldots, l, \\
I_{s_{1}} \cap I_{s_{2}}=\varnothing, \quad 1 \leq s_{1}<s_{2} \leq l,
\end{gathered}
$$

где $I_{s}$ обозначает множество существенных аргументов функции $\varphi_{s}$, $s=1, \ldots, l$.

Теорема 4. Пусть $K=G F(q)$ - простое поле, $\left\{f_{1}(x), \ldots, f_{m}(x)\right\} \supseteq Q_{n, l}$, $l \leq m$. Тогда

$$
\rho\left(\left(K^{n}\right)_{f}\right) \leq \prod_{s=1}^{l}\left(1-q^{-2\left|I_{s}\right|}\right) .
$$

Замечание 2. Теоремы 1 и 2 можно применить к системе включений (6), используя оценки (24) и (25), если множество $H_{f}$ (множество $\left.\left(K^{n}\right)_{f}\right)$ не содержит подобных векторов (в случае $K=G F(2)$ они заведомо отсутствуют). При $q \geq 3$ это условие выполнено, если, например, 
выполнено хотя бы одно из включений $\left\{f_{1}(x), \ldots, f_{m}(x)\right\} \supseteq Q^{(2)}\left[x_{1}, \ldots, x_{n}\right]$ или $\left\{f_{1}(x), \ldots, f_{m}(x)\right\} \supseteq Q^{\left(t_{1}\right)}\left[x_{1}, \ldots, x_{n}\right] \cup Q^{\left(t_{2}\right)}\left[x_{1}, \ldots, x_{n}\right], 2<t_{1}<t_{2}$, НОД $\left(t_{1}-1, t_{2}-1\right)=1$, где

$$
Q^{(t)}\left[x_{1}, \ldots, x_{n}\right]=\bigcup_{\substack{d_{1}, \ldots, d_{n} \in\{0, q-1\} \\ d_{1}+\ldots+d_{n}=t}}\left\{x_{1}^{d_{1}} \ldots x_{n}^{d_{n}}\right\}
$$

- множество всех произведений переменных из набора $\left\{x_{1}, \ldots, x_{n}\right\}$ степени $t$. Достаточность приведенных условий легко доказать, принимая во внимание тот факт, что $Q^{(t)}\left[x_{1}, \ldots, x_{n}\right] \supset\left\{x_{1}^{t}, \ldots, x_{n}^{t}\right\}$.

Следующая теорема дает нижнюю оценку величины $\rho\left(\left(K^{n}\right)_{f}\right)$ в случае, когда $K=G F\left(2^{\alpha}\right)$ - конечное поле характеристики два.

Теорема 5. Пусть $K=G F(q)$, где $q=2^{\alpha}, \alpha \geq 1$. Тогда при любом $n \geq 1$ и любом отображении $f: K^{n} \rightarrow K^{m}, m \geq 1$, выполнено неравенство

$$
\rho\left(\left(K^{n}\right)_{f}\right) \geq 3 \cdot q^{-n}-2 \cdot q^{-2 n}
$$

Далее показывается, что оценка (27) достижима при $q=2$.

Теорема 6. Пусть $K=G F(2), n \geq 2, m \geq C_{n}^{2}, f=\left(f_{1}, \ldots, f_{m}\right)$, где

$$
\left\{f_{1}(x), \ldots, f_{m}(x)\right\} \supseteq\left\{x_{i} x_{j}: 1 \leq i<j \leq n\right\} .
$$

Тогда

$$
\rho\left(\left(K^{n}\right)_{f}\right)=3 \cdot 2^{-n}-2^{1-2 n}
$$

В статье также доказаны оценки величины $\rho\left(\left(K^{n}\right)_{f}\right)$ в случае, когда множество координатных функций отображения $f$ содержит как все попарные произведения переменных $x_{1}, \ldots, x_{n}$, так и сами эти переменные во второй степени. Для поля характеристики два получена следующая оценка.

Теорема 7. Пусть $K=G F(q), q=2^{\alpha}, \alpha \geq 2, n \geq 2, m \geq n+C_{n}^{2}$, $f=\left(f_{1}, \ldots, f_{m}\right)$, при этом

$$
\left\{f_{1}(x), \ldots, f_{m}(x)\right\} \supseteq\left\{x_{i} x_{j}: 1 \leq i \leq j \leq n\right\} .
$$

Тогда

$$
\rho\left(\left(K^{n}\right)_{f}\right) \leq(q+1) q^{-n}-q^{1-2 n} .
$$

Для поля характеристики, отличной от двух, получена следующая оценка. 
Теорема 8. Пусть $K=G F(q), q=p^{\alpha}, p \geq 3, \alpha \geq 1, n \geq 2, m \geq n+C_{n}^{2}$, $f=\left(f_{1}, \ldots, f_{m}\right)$ и выполняется условие (30). Тогда

$$
\rho\left(\left(K^{n}\right)_{f}\right) \leq(n+1) q^{-n}
$$

Приведем примеры, в которых используются следствие 1 и теоремы 4, $6,7,8$.

Пример 1. Пусть $K=G F(2),\left\{f_{1}(x), \ldots, f_{m}(x)\right\} \supseteq\left\{x_{1} x_{2}, \ldots, x_{l-1} x_{l}\right\}$, где $l=[n / 2]$. Положим $D=V^{n} \backslash\left\{0^{n}\right\}, n \geq 200,|B|=1, T=n$. При $n \geq 200$, согласно формуле (25), имеем $\rho\left(\left(K^{n}\right)_{f}\right) \leq(15 / 16)^{100}=$ $=0,0001$. Используя (16)-(18), получаем следующие значения правых частей неравенств (15):

\begin{tabular}{|r|c|c|c|c|c|}
\hline$r=$ & 0 & 1 & 2 & 3 & 4 \\
\hline$\tilde{\lambda}^{r} e^{-\tilde{\lambda}} / r !=$ & $0,368 \ldots$ & $0,368 \ldots$ & $0,184 \ldots$ & $0,061 \ldots$ & $0,015 \ldots$ \\
\hline$\left|\mathbf{P}\{\xi=r\}-\tilde{\lambda}^{r} e^{-\tilde{\lambda}} / r !\right| \leq$ & $2,9 \cdot 10^{-5}$ & $1,0 \cdot 10^{-4}$ & $1,4 \cdot 10^{-4}$ & $9,1 \cdot 10^{-5}$ & $3,4 \cdot 10^{-5}$ \\
\hline
\end{tabular}

Здесь $\xi=\xi\left(H_{f}, A, S^{\prime}, B\right)$. Отметим, что основной вклад в значения правых частей $(15)$ вносят величины $S_{3}(r)$. В нашем случае $\tilde{\lambda}=1-2^{-n}<1$. Поэтому величины $S_{3}(r)$ мы оценили по формулам

$$
\begin{gathered}
S_{3}(0) \leq\left. S_{3}(0)\right|_{\tilde{\lambda}=1}=\rho\left(\left(K^{n}\right)_{f}\right)(3-e), \\
S_{3}(1) \leq\left. S_{3}(1)\right|_{\tilde{\lambda}=1}=\rho\left(\left(K^{n}\right)_{f}\right), \\
S_{3}(r) \leq\left. S_{3}(r)\right|_{\tilde{\lambda}=1}=\rho\left(\left(K^{n}\right)_{f}\right) \frac{e(r-1)}{r !}, \quad r \geq 2 .
\end{gathered}
$$

Пример 2. Пусть $K=G F(2)$ и выполнено условие (28). Положим $D=$ $=K^{n} \backslash\left\{0^{n}\right\}, n \geq 30,|B|=1, T=n$. В этом случае, согласно (29), имеем $\rho\left(\left(K^{n}\right)_{f}\right) \leq 3 \cdot 2^{-n} \leq 3 \cdot 10^{-9}$. Получим следующие значения:

\begin{tabular}{|r|c|c|c|c|c|}
\hline$r=$ & 0 & 1 & 2 & 3 & 4 \\
\hline$\tilde{\lambda}^{r} e^{-\tilde{\lambda}} / r !=$ & $0,368 \ldots$ & $0,368 \ldots$ & $0,184 \ldots$ & $0,061 \ldots$ & $0,015 \ldots$ \\
\hline$\left|\mathbf{P}\{\xi=r\}-\tilde{\lambda}^{r} e^{-\tilde{\lambda}} / r !\right| \leq$ & $3,6 \cdot 10^{-9}$ & $8,5 \cdot 10^{-9}$ & $8,2 \cdot 10^{-9}$ & $4,6 \cdot 10^{-9}$ & $1,6 \cdot 10^{-9}$ \\
\hline
\end{tabular}

В данном случае основной вклад в значения правых частей (15) вносят величины $S_{2}(r), S_{3}(r)$.

Отметим, что в примере 2, где использованы теорема 6 и условие $n \geq 30$, верхние границы для значений $\left|\mathbf{P}\{\xi=r\}-\tilde{\lambda}^{r} e^{-\tilde{\lambda}} / r !\right|$ на четыре порядка меньше соответствующих границ из примера 1 , где были использованы теорема 4 и условие $n \geq 200$. 
Пример 3. Пусть $K=G F(q), q \geq 3$, и выполнено условие (30). Значения остальных параметров оставим такими же, как в примере 2 . Согласно (31) и (32) имеем $\rho\left(\left(K^{n}\right)_{f}\right) \leq 1,6 \cdot 10^{-13}$. Получим

\begin{tabular}{|r|c|c|c|c|c|}
\hline$r=$ & 0 & 1 & 2 & 3 & 4 \\
\hline$\tilde{\lambda}^{r} e^{-\tilde{\lambda}} / r !=$ & $0,368 \ldots$ & $0,368 \ldots$ & $0,184 \ldots$ & $0,061 \ldots$ & $0,015 \ldots$ \\
\hline$\left|\mathbf{P}\{\xi=r\}-\tilde{\lambda}^{r} e^{-\tilde{\lambda}} / r !\right| \leq$ & $6 \cdot 10^{-14}$ & $1,9 \cdot 10^{-13}$ & $2,4 \cdot 10^{-13}$ & $1,6 \cdot 10^{-13}$ & $6 \cdot 10^{-14}$ \\
\hline
\end{tabular}

В этом примере, как и в примере 2, основной вклад в значения правых частей (15) вносят величины $S_{2}(r), S_{3}(r)$.

\section{3. Доказательство теоремы 2}

Введем обозначения

$$
J=\bigcup_{x \in D}\{(x, b): b+S(x) \in B\}, \quad \xi=\xi(D, A, S, B) .
$$

Положим, (используя обозначение $\left.v^{i}=\left(x^{i}, b^{i}\right)\right)$

$$
J_{k}=\left\{\left(v^{1}, \ldots, v^{k}\right) \in J^{k}: v^{\alpha} \neq v^{\beta}(\alpha \neq \beta)\right\} .
$$

Воспользуемся формулами метода включения-исключения (см., например, $[5$, гл.IV] или $[6$, гл. $1, \S 1$, п.4]). При любой реализации отображения $S$ получим:

$$
\mathbf{P}\{\xi=r\}=\sum_{k=r}^{|J|}(-1)^{k-r} C_{k}^{r} B_{k}, \quad r=0,1, \ldots,
$$

где

$$
B_{k}=\mathbf{E} C_{\xi}^{k}=\frac{1}{k !} \sum_{\left(v^{1}, \ldots, v^{k}\right) \in J_{k}} \mathbf{P}\left\{A x^{1}=b^{1}, \ldots, A x^{k}=b^{k}\right\}
$$

Из (33) и неравенств Бонферрони следует, что

$$
\mathbf{P}\{\xi=r\}=\sum_{k=r}^{N}(-1)^{k-r} C_{k}^{r} B_{k}+R_{N+1},
$$

где $0 \leq r \leq N \leq|J|-1$, a

$$
\left|R_{N+1}\right| \leq C_{N+1}^{r} B_{N+1} .
$$


Далее будут использованы некоторые выкладки из доказательства теоремы 4 работы [4].

Лемма 1. Пусть $x^{1}, \ldots, x^{k} \in K^{n}, b^{1}, \ldots, b^{k} \in K^{T}$ и выполнено равенство $\Delta=0$. Тогда при всех $k=1,2, \ldots$

$$
\mathbf{P}\left\{A x^{1}=b^{1}, \ldots, A x^{k}=b^{k}\right\} \leq\left(\frac{1}{q}\right)^{j T}
$$

если $1 \leq \operatorname{rank}\left(x^{1}, \ldots, x^{k}\right)=j \leq k-1$, а если $\operatorname{rank}\left(x^{1}, \ldots, x^{k}\right)=k$, то

$$
\mathbf{P}\left\{A x^{1}=b^{1}, \ldots, A x^{k}=b^{k}\right\}=\left(\frac{1}{q}\right)^{k T} .
$$

Эта лемма вытекает из леммы 1 в [4].

Положим $D_{1}=\varnothing, I_{1}=\varnothing$, а при $k \geq 2$,

$$
\begin{gathered}
D_{k, j}=\left\{\left(x^{1}, \ldots, x^{k}\right) \in D^{k}: \operatorname{rank}\left(x^{1}, \ldots, x^{k}\right)=j\right\}, \quad D_{k}=\bigcup_{j=1}^{k-1} D_{k, j}, \\
I_{k}=\left\{\left(\left(x^{1}, b^{1}\right), \ldots,\left(x^{k}, b^{k}\right)\right) \in J_{k}:\left(x^{1}, \ldots, x^{k}\right) \in D_{k}\right\} .
\end{gathered}
$$

Используя множество $I_{k}$, представим величину $B_{k} k$ ! в виде суммы

$$
B_{k} k !=\Sigma_{1, k}+\Sigma_{2, k}
$$

где

$$
\begin{aligned}
\Sigma_{1, k} & =\sum_{\left(v^{1}, \ldots, v^{k}\right) \in J_{k} \backslash I_{k}} \mathbf{P}\left\{A x^{1}=b^{1}, \ldots, A x^{k}=b^{k}\right\}, \\
\Sigma_{2, k} & =\sum_{\left(v^{1}, \ldots, v^{k}\right) \in I_{k}} \mathbf{P}\left\{A x^{1}=b^{1}, \ldots, A x^{k}=b^{k}\right\} .
\end{aligned}
$$

Оценим величины $B_{k} k$ ! сверху. Начнем с (41). В [4] было показано, что $\Sigma_{2,1}=\Sigma_{2,2}=0$ (см. формулу (31) в [4]), а при $k \geq 3$ выполнено неравенство (см. выкладки, следующие после доказательства леммы 3 в [4])

$$
\Sigma_{2, k} \leq \rho(J) \sum_{j=2}^{k-1}\left(\frac{|J|}{q^{T}}\right)^{j}=\rho(J) \sum_{j=2}^{k-1} \tilde{\lambda}^{j}, \quad k \geq 3,
$$

где согласно лемме 4 из [4]

$$
\rho(J) \leq \rho(D) \rho(B)
$$


Оценим теперь величину (40). Используя формулу (38), получаем, что при всех $\left(v^{1}, \ldots, v^{k}\right) \in J_{k} \backslash I_{k}$ выполнено равенство

$$
\mathbf{P}\left\{A x^{1}=b^{1}, \ldots, A x^{k}=b^{k}\right\}=\prod_{j=1}^{k} \mathbf{P}\left\{A x^{j}=b^{j}\right\}=\left(\frac{1}{q}\right)^{k T} .
$$

Поэтому $\Sigma_{1, k} \leq \tilde{\lambda}^{k}, k \geq 2$. Из приведенных оценок, в частности, из (39), (42) и (43), получаем соотношения

$$
\begin{gathered}
B_{0}=1, \quad B_{1}=\tilde{\lambda}^{j}, \quad 2 B_{2} \leq \tilde{\lambda}^{2}, \\
B_{k} k ! \leq \tilde{\lambda}^{k}+\rho(D) \rho(B) \sum_{j=2}^{k-1} \tilde{\lambda}^{j}, \quad k \geq 3 .
\end{gathered}
$$

Сразу отметим, что из (36), (45) и (46) следует оценка для остаточного члена в (35):

$$
\left|R_{N+1}\right| \leq \frac{\tilde{\lambda}^{N+1}}{r !(N+1-r) !}+\rho(D) \rho(B) \sum_{j=2}^{N} \frac{\tilde{\lambda}^{j}}{r !(N+1-r) !} .
$$

Оценим величины $B_{k} k$ ! снизу. Используя (44), получаем

$$
\Sigma_{1, k}=\sum_{\left(v^{1}, \ldots, \nu^{k}\right) \in J_{k} \backslash I_{k}} \prod_{j=1}^{k} \mathbf{P}\left\{A x^{j}=b^{j}\right\}=\tilde{\lambda}^{k}-\Sigma_{1, k}^{1}-\Sigma_{1, k}^{2},
$$

где

$$
\begin{gathered}
\Sigma_{1, k}^{1}=\sum_{\left(v^{1}, \ldots, v^{k}\right) \in I_{k}} \prod_{j=1}^{k} \mathbf{P}\left\{A x^{j}=b^{j}\right\}, \\
\Sigma_{1, k}^{2}=\sum_{\left(v^{1}, \ldots, v^{k}\right)}^{\prime} \prod_{j=1}^{k} \mathbf{P}\left\{A x^{j}=b^{j}\right\},
\end{gathered}
$$

и последняя сумма берется по всем таким наборам индексов $\left(v^{1}, \ldots, v^{k}\right)$, в которых имеется хотя бы одна пара равных. Нетрудно убедиться, что (см. оценку (3.16) в [7])

$$
\Sigma_{1, k}^{2} \leq \frac{k}{q^{T}} \tilde{\lambda}^{k-1} .
$$


Вместе с этим (см. (28) и замечание 2 в [4])

$$
\Sigma_{1, k}^{1} \leq \tilde{\lambda}^{k} \frac{1}{|D|^{k}} \sum_{j=1}^{k-1}\left|D_{k, j}\right|
$$

Заметим, что $\left|D_{k, 1}\right|=0, k=2,3, \ldots$ Для величин $\left|D_{k, j}\right|$ при $k \geq 3,2 \leq j \leq k-$ - 1 в [4] получены оценки (19). Здесь мы воспользуемся более точными оценками.

Лемма 2. Пусть множество $D$ не содержит подобных векторов, тогда при $k \geq 3,2 \leq j \leq k-1$

$$
\left|D_{k, j}\right| \leq C_{k}^{j} C_{j}^{2}(|D| q)^{j-2}|D|^{2} \rho(D)
$$

Доказательство. Имеем

$$
\begin{gathered}
\left|D_{k, j}\right| \leq C_{k}^{j} \mid\left\{\left(x^{1}, \ldots, x^{k}\right) \in D^{k}\right. \\
\left.\operatorname{rank}\left(x^{1}, \ldots, x^{j}\right)=j, \operatorname{rank}\left(x^{1}, \ldots, x^{j}, x^{l}\right)=j, l=j+1, \ldots, k\right\} \mid \\
\leq C_{k}^{j} \mid\left\{\left(x^{1}, \ldots, x^{j+1}\right) \in D^{j+1}\right. \\
\left.\operatorname{rank}\left(x^{1}, \ldots, x^{j}\right)=j, \operatorname{rank}\left(x^{1}, \ldots, x^{j}, x^{j+1}\right)=j\right\} \mid \\
\leq C_{k}^{j} C_{j}^{2} \mid\left\{\left(x^{1}, \ldots, x^{j+1}\right) \in D^{j+1}:\right. \\
\left.x^{j+1}=a_{1} x^{1}+a_{2} x^{2}+a_{3} x^{3}+\ldots+a_{j} x^{j}, a_{1}, a_{2} \in K \backslash\{0\}, a_{3}, \ldots, a_{j} \in K\right\} \mid .
\end{gathered}
$$

Из (52) и определения величины $\rho(D)$ получаем (51). Лемма 2 доказана.

Из (50), (51) получаем

$$
\begin{aligned}
& \Sigma_{1, k}^{1} \leq \rho(D) \tilde{\lambda}^{k} \sum_{j=2}^{k-1} C_{k}^{j}|D|^{-(k-j)} C_{j}^{2} q^{j-2} \\
& \leq \rho(D) \tilde{\lambda}^{k} C_{k}^{2}\left[\left(q+|D|^{-1}\right)^{k-2}-q^{k-2}\right] .
\end{aligned}
$$

Наконец, отметим, что (см. (35), (39), (48))

$$
\sum_{k=r}^{N}(-1)^{k-r} C_{k}^{r} \frac{\tilde{\lambda}^{k}}{k !}=\frac{\tilde{\lambda}^{r}}{r !} \sum_{k=r}^{N} \frac{(-\tilde{\lambda})^{k-r}}{(k-r) !}=\frac{\tilde{\lambda}^{r}}{r !} e^{-\tilde{\lambda}}+\tilde{R}_{N+1}
$$


где

$$
\tilde{R}_{N+1}=-\frac{\tilde{\lambda}^{r}}{r !} \sum_{k=N+1}^{\infty} \frac{(-\tilde{\lambda})^{k-r}}{(k-r) !}
$$

По условию теоремы $N-r>\tilde{\lambda}$. Значит, величины $\tilde{\lambda}^{k-r} /(k-r)$ ! при $k \geq N+1$ строго убывают с ростом $k$ (и стремятся к нулю). Поэтому $\left|\tilde{R}_{N+1}\right|$ меньше абсолютной величины первого члена ряда (55) (см. теорему Лейбница для знакопеременных рядов и замечание к ней в [8, раздел 381]):

$$
\left|\tilde{R}_{N+1}\right| \leq \frac{\tilde{\lambda}^{N+1}}{r !(N+1-r) !}
$$

С помощью неравенств (35), (39), (48), (54) получаем

$$
\left|\mathbf{P}\{\xi=r\}-\frac{\tilde{\lambda}^{r}}{r !} e^{-\tilde{\lambda}}\right| \leq\left|\tilde{R}_{N+1}\right|+\left|R_{N+1}\right|+\sum_{k=r}^{N} \frac{C_{k}^{r}}{k !}\left(\Sigma_{1, k}^{1}+\Sigma_{1, k}^{2}+\Sigma_{2, k}\right) .
$$

Используя (42), (43), (50), (49), (47), (56), приходим к оценкам

$$
\begin{gathered}
\sum_{k=r}^{N} \frac{C_{k}^{r}}{k !} \Sigma_{1, k}^{i} \leq S_{i, N}(r), \quad i=1,2, \\
\left|\tilde{R}_{N+1}\right|+\left|R_{N+1}\right|+\sum_{k=r}^{N} \frac{C_{k}^{r}}{k !} \Sigma_{2, k} \leq \frac{2 \tilde{\lambda}^{N+1}}{r !(N+1-r) !}+S_{3, N}(r) .
\end{gathered}
$$

Из этих неравенств и (57) вытекает (9). Теорема 2 доказана.

\section{4. Доказательство теоремы 4}

Напомним, что $\left(K^{n}\right)_{f}$ является образом множества $K^{n}$ в $K^{n+m}$ при отображении

$$
x=\left(x_{1}, \ldots, x_{n}\right) \longrightarrow y=(x, f(x))=\left(x_{1}, \ldots, x_{n}, f_{1}(x), \ldots, f_{m}(x)\right) .
$$

Согласно определению

$$
\rho\left(\left(K^{n}\right)_{f}\right)=\frac{N_{f}}{q^{2 n}}
$$

где $N_{f}$ - максимально возможное при $a_{1}, a_{2}, a_{3} \in K \backslash\{0\}, c \in K^{n+m}-$ число решений уравнения $a_{1} y^{1}+a_{2} y^{2}+a_{3} y^{3}=c$ в $K^{n+m}$ относительно тройки векторов $\left(y^{1}, y^{2}, y^{3}\right) \in\left(\left(K^{n}\right)_{f}\right)^{3}$. Заметим, что одно из чисел $a_{1}, a_{2}, a_{3} \in K \backslash\{0\}$ 
можно положить равным единице. Взяв $a_{3}=1$, перепишем это уравнение относительно переменных $x_{i}^{1}, x_{i}^{2}, x_{i}^{3}, i=1, \ldots, n$, как объединение двух систем уравнений: системы из $n$ уравнений

$$
a_{1} x_{i}^{1}+a_{2} x_{i}^{2}+x_{i}^{3}=c_{i}^{1}, \quad i=1, \ldots, n,
$$

и системы из $m$ уравнений

$$
a_{1} f_{s}\left(x^{1}\right)+a_{2} f_{s}\left(x^{2}\right)+f_{s}\left(x^{3}\right)=c_{s}^{2}, \quad s=1, \ldots, m .
$$

В данном случае $c=\left(c^{1}, c^{2}\right)=\left(c_{1}^{1}, \ldots, c_{n}^{1}, c_{1}^{2}, \ldots, c_{m}^{2}\right)$.

Не нарушая общности рассуждений, будем рассматривать функции $\varphi_{1}(x), \ldots, \varphi_{l}(x)$ из множества $Q_{n, l}$ в качестве первых $l$ координатных функций $f_{1}(x), \ldots, f_{l}(x)$ отображения $f(x)=\left(f_{1}(x), \ldots, f_{m}(x)\right)$. Заметим, что согласно условию теоремы $m \geq l=\left|Q_{n, l}\right|$.

Исключив из второй системы последние $m-l$ уравнений и оставив в ней лишь уравнения, соответствующие функциям из множества $Q_{n, l}$, получим систему:

$$
a_{1} f_{s}\left(x^{1}\right)+a_{2} f_{s}\left(x^{2}\right)+f_{s}\left(x^{3}\right)=c_{s}^{2}, \quad s=1, \ldots, l .
$$

Максимальное число решений системы (58)-(59) обозначим $N_{f}^{*}$. Очевидно, что

$$
N_{f}^{*} \geq N_{f}
$$

Система (58)-(59) состоит из $n+l$ уравнений относительно $3 n$ неизвестных элементов $x_{i}^{1}, x_{i}^{2}, x_{i}^{3}, i=1, \ldots, n$, поля $K$. Ее уравнения можно распределить по $l+1$ системам следующим образом. Первые $l$ систем имеют вид (здесь $s=1, \ldots, l$ )

$$
\begin{gathered}
a_{1} \varphi_{s}\left(x_{i}^{1}, i \in I_{s}\right)+a_{2} \varphi_{s}\left(x_{i}^{2}, i \in I_{s}\right)+\varphi_{s}\left(x_{i}^{3}, i \in I_{s}\right)=c_{s}^{2}, \\
a_{1} x_{i}^{1}+a_{2} x_{i}^{2}+x_{i}^{3}=c_{i}^{1}, \quad i \in I_{s} .
\end{gathered}
$$

Напомним, что $I_{s}-$ множество существенных аргументов функции $\varphi_{s}(x)$. Поэтому выше использована запись $\varphi_{s}(x)=\varphi_{s}\left(x_{i}, i \in I_{s}\right)$.

Система с номером $(l+1)$ состоит из $n-\left|I_{1}\right|-\ldots-\left|I_{l}\right|$ уравнений системы (58), не вошедших в первые $l$ систем.

Обозначим через $N_{f}^{(s)}$ максимальное (по всем допустимым вариантам выбора коэффициентов и правых частей уравнений) число решений $s$-й системы. Так как множества неизвестных, входящих в разные системы, попарно не пересекаются, то

$$
N_{f}^{*}=\prod_{s=1}^{l+1} N_{f}^{(s)}
$$


Ниже мы покажем, что

$$
N_{f}^{(s)} \leq q^{2\left|I_{s}\right|}-1, \quad s=1, \ldots, l .
$$

Система с номером $(l+1)$ позволяет выразить элементы $x_{i}^{3}$ через $x_{i}^{1}$ и $x_{i}^{2}$ для всех $i \in\{1, \ldots, n\} \backslash\left(I_{1} \cup \ldots \cup I_{l}\right)$, что приводит к равенству

$$
N_{f}^{(l+1)}=q^{2\left(n-\left|I_{1}\right|-\ldots-\left|I_{l}\right|\right)} .
$$

Из (61)-(63) и (60) следуют неравенства

$$
N_{f} \leq N_{f}^{*} \leq q^{2 n} \prod_{s=1}^{l}\left(1-q^{-2\left|I_{s}\right|}\right) .
$$

Поэтому

$$
\rho\left(\left(V^{n}\right)_{f}\right) \leq \prod_{s=1}^{l}\left(1-q^{-2\left|I_{s}\right|}\right),
$$

что и требовалось доказать. Итак, для завершения доказательства теоремы 4 надо убедиться в справедливости неравенств (62).

Рассмотрим $s$-ю систему при произвольном $1 \leq s \leq l$. Используя линейные уравнения, выразим неизвестные $x_{i}^{3}$ через $x_{i}^{1}$ и $x_{i}^{2}$ и подставим их в нелинейное уравнение. Полагая $\left(x_{i}^{1}, i \in I_{S}\right)=y,\left(x_{i}^{2}, i \in I_{S}\right)=z$, $\left(c_{i}^{1}, i \in I_{S}\right)=\bar{c}_{s}^{1}$, получаем уравнение в $K$

$$
a_{1} \varphi_{s}(y)+a_{2} \varphi_{s}(z)+\varphi_{s}\left(\bar{c}_{s}^{1}-a_{1} y-a_{2} z\right)=c_{s}^{2} .
$$

Нам достаточно показать, что выражение в левой части (65) как функция от $y$ и $z$ не является тождественной константой. Действительно, тогда найдется пара $(y, z) \in\left(K^{\left|I_{s}\right|}\right)^{2}$, не удовлетворяющая уравнению (65). Значит, это уравнение имеет не более $q^{2\left|I_{s}\right|}-1$ решений.

Пусть множество $I_{s}=\left\{x_{j_{1}}, \ldots, x_{j_{k}}\right\}$ при некоторых $1 \leq k<n$ и $1 \leq$ $\leq j_{1}<\ldots<j_{k} \leq n$. Тогда функция $\varphi_{s}(x)$ может быть выражена формулой (суммирование производится в поле $K$ )

$$
\varphi_{s}(x)=\sum_{\substack{d_{1}, \ldots, d_{k} \in\{0, \ldots, q-1\} \\ 0 \leq d_{1}+\ldots+d_{k} \leq d}} \omega_{d_{1}, \ldots, d_{k}}^{(s)} \prod_{u=1}^{k} x_{j_{u}}^{d_{u}} .
$$

Согласно условиям теоремы 4, правая часть равенства (66) является многочленом степени $d \geq 2$. 
Подставим (66) в (65) и опустим в разложении левой части (65) члены степени меньше $d$. Получим выражение

$$
\sum_{\substack{d_{1}, \ldots, d_{k} \in\{0, \ldots, q-1\} \\ d_{1}+\ldots+d_{k}=d}} \omega_{d_{1}, \ldots, d_{k}}^{(s)} \Phi_{d_{1}, \ldots, d_{k}}(y, z),
$$

где

$$
\begin{gathered}
\Phi_{d_{1}, \ldots, d_{k}}(y, z)=a_{1} \prod_{u=1}^{k} y_{j_{u}}^{d_{u}}+a_{2} \prod_{u=1}^{k} z_{j_{u}}^{d_{u}}+(-1)^{d} \prod_{u=1}^{k}\left(a_{1} y_{j_{u}}+a_{2} z_{j_{u}}\right)^{d_{u}}= \\
=\left(a_{1}+\left(-a_{1}\right)^{d}\right) \prod_{u=1}^{k} y_{j_{u}}^{d_{u}}+\left(a_{2}+\left(-a_{2}\right)^{d}\right) \prod_{u=1}^{k} z_{j_{u}}^{d_{u}}+ \\
+(-1)^{d} \sum_{i_{1}, \ldots, i_{k}} \prod_{u=1}^{k} C_{d_{u}}^{i_{u}}\left(a_{1} y_{j_{u}}\right)^{i_{u}}\left(a_{2} z_{j_{u}}\right)^{d_{u}-i_{u}} .
\end{gathered}
$$

Отметим три важных свойства, которые будут использованы далее.

1) Все выражения $\prod_{u=1}^{k} y_{j_{u}}^{d_{u}}, \prod_{u=1}^{k} z_{j_{u}}^{d_{u}}, \prod_{u=1}^{k} C_{d_{u}}^{i_{u}}\left(a_{1} y_{j_{u}}\right)^{i_{u}}\left(a_{2} z_{j_{u}}\right)^{d_{u}-i_{u}}$ из всех функций $\Phi_{d_{1}, \ldots, d_{k}}(y, z)$ содержат разные наборы степеней у переменных (как из множества $\left\{y_{j_{1}}, \ldots, y_{j_{k}}\right\}$, так и из множества $\left\{z_{j_{1}}, \ldots, z_{j_{k}}\right\}$ ). Поэтому они могут не войти в сумму (67) только при наличии нулевых коэффициентов перед ними.

2) Сумма $\sum_{i_{1}, \ldots, i_{k}}$ содержит хотя бы одно ненулевое слагаемое. Действительно, число слагаемых в ней равно $\left(d_{1}+1\right) \cdot \ldots \cdot\left(d_{k}+1\right)-2$. Это число отлично от нуля, поскольку $d_{1}+\ldots+d_{k} \geq 2$. Все величины $C_{d_{u}}^{i_{u}}$ в ней не делятся на простое $q$ при $d_{u} \in\{0, \ldots, q-1\}$ (напомним, что $K \stackrel{d_{u}}{=} G F(q)-$ простое поле). Значит, все мономы, входящие в сумму $\sum_{i_{1}, \ldots, i_{k}}$, имеют ненулевые коэффициенты. Следовательно, все функции $\Phi_{d_{1}, \ldots, d_{k}}(y, z)$ отличны от нуля.

3) Среди коэффициентов $\omega_{d_{1}, \ldots, d_{k}}^{(s)}$ найдется коэффициент $\omega_{d_{1}^{*}, \ldots, d_{k}^{*}}^{(s)} \neq 0$ при некоторых $d_{1}^{*}, \ldots, d_{k}^{*} \in\{0, \ldots, q-1\}, d_{1}^{*}+\ldots+d_{k}^{*}=d$.

Согласно 3), достаточно рассмотреть функцию $\Phi_{d_{1}^{*}, \ldots, d_{k}^{*}}(y, z)$ и убедиться, что она отлична от константы. А это следует из свойств 1) и 2).

Значит, выражение (67) и левая часть уравнения (65) отличны от констант. Следовательно, выполнены неравенства (62). Доказательство теоремы 4 завершено. 


\section{5. Доказательства теорем 5-8}

Доказательство теоремы 5. Пусть $K=G F(q)$, где $q=2^{\alpha}$. Согласно определению

$$
\rho\left(\left(K^{n}\right)_{f}\right)=\frac{N_{f}}{q^{2 n}},
$$

где $N_{f}$ - максимально возможное при $a_{1}, a_{2} \in K \backslash\{0\},\left(c^{1}, c^{2}\right) \in K^{n+m}$ число решений системы уравнений

$$
\begin{gathered}
a_{1} x^{1}+a_{2} x^{2}+x^{3}=c^{1}, \\
a_{1} f\left(x^{1}\right)+a_{2} f\left(x^{2}\right)+f\left(x^{3}\right)=c^{2},
\end{gathered}
$$

относительно тройки векторов $\left(x^{1}, x^{2}, x^{3}\right) \in\left(K^{n}\right)^{3}$. Используя линейное уравнение (70) этой системы, выразим вектор $x^{3}$ через $x^{1}, x^{2}$ и подставим в уравнение (71). Получим

$$
a_{1} f\left(x^{1}\right)+a_{2} f\left(x^{2}\right)+f\left(c^{1}-a_{1} x^{1}-a_{2} x^{2}\right)=c^{2} .
$$

Очевидно, что уравнение (72) имеет такое же число решений, как и система (70)-(71).

Положим $a_{1}=a_{2}=1, c^{2}=f\left(c^{1}\right)$. При этом условии все пары $n$-мерных векторов $x^{1}, x^{2}$, удовлетворяющие равенству $x^{1}=x^{2}$ (и, следовательно, равенству $x^{1}+x^{2}=0^{n}$ ), являются решениями уравнения (72). Их число $N^{(1)}=$ $=q^{n}$. Кроме того, согласно условиям $a_{1}=a_{2}=1, c^{2}=f\left(c^{1}\right)$ и равенствам $f(x)+f(-x)=0^{m}$ для всех $x \in K^{n}$ решениями уравнения (72) являются все пары $x^{1}, x^{2}$ вида

$$
x^{1}=c^{1}, \quad x^{2} \in K^{n} \backslash\left\{c^{1}\right\}
$$

и все пары $x^{1}, x^{2}$ вида

$$
x^{1} \in K^{n} \backslash\left\{c^{1}\right\}, \quad x^{2}=c^{1} .
$$

Общее число таких пар $N^{(2)}=2\left(q^{n}-1\right)$. Следовательно,

$$
N_{f} \geq N^{(1)}+N^{(2)}=3 \cdot q^{n}-2 .
$$

Очевидно, что неравенство (73) выполняется при любом $n \geq 1$ и любом отображении $f: K^{n} \rightarrow K^{m}, m \geq 1$, где $K-$ конечное поле характеристики два. Из (69), (73) следует неравенство (27). Теорема 5 доказана.

Доказательство теоремы 6. Пусть $\varphi(x)=\left(\varphi_{1}(x), \ldots, \varphi_{m}(x)\right)$ - произвольное отображение из $K^{n}$ в $K^{m}$. Обозначим $M[\varphi(x)]$ множество прообразов элемента $\varphi(x)$. 
Лемма 3. Пусть $K=G F(q), q=2^{\alpha}, n \geq 2, m=C_{n}^{2}, f^{(n)}=\left(f_{1}, \ldots, f_{C_{n}^{2}}\right)$, где

$$
\left\{f_{1}(x), \ldots, f_{C_{n}^{2}}(x)\right\}=Q^{(2)}\left[x_{1}, \ldots, x_{n}\right]=\left\{x_{i} x_{j}: 1 \leq i<j \leq n\right\} .
$$

Тогда при $b \neq 0^{n}$ справедливы неравенства

$$
\left|M\left[f^{(n)}(x)+f^{(n)}(b+x)\right]\right| \leq q \quad \forall x \in K^{n} .
$$

Доказательство леммы проведем с использованием индукции по параметру $n \geq 2$ и формул

$$
\begin{gathered}
Q^{(2)}\left[x_{1}, x_{2}\right]=\left\{x_{1} x_{2}\right\}, \\
Q^{(2)}\left[x_{1}, \ldots, x_{n}\right]=Q^{(2)}\left[x_{1}, \ldots, x_{n-1}\right]+\bigcup_{i=1}^{n-1}\left\{x_{i} x_{n}\right\}, \quad n \geq 3 .
\end{gathered}
$$

Сначала убедимся, что неравенства (75) выполняются при $n=2$. В этом случае $f^{(2)}(x)=x_{1} x_{2}$, и при заданном $x=\left(x_{1}, x_{2}\right)$ уравнение $f^{(2)}(\tilde{x})+f^{(2)}(b+$ $+\tilde{x})=f^{(2)}(x)+f^{(2)}(b+x)$ относительно $\tilde{x}=\left(\tilde{x}_{1}, \tilde{x}_{2}\right)$ имеет вид

$$
b_{1} \tilde{x}_{2}+b_{2} \tilde{x}_{1}=b_{1} x_{2}+b_{2} x_{1} .
$$

Пусть $b_{1} \neq 0$, тогда при любом значении $\tilde{x}_{1} \in G F(q)$ уравнение (78) имеет для $\tilde{x}_{2}=b_{1}^{-1}\left(b_{2} \tilde{x}_{1}+b_{1} x_{2}+b_{2} x_{1}\right)$ единственное решение. Значит, в этом случае $\left|M\left[f^{(2)}(x)+f^{(2)}(b+x)\right]\right|=q$. То же самое получаем при $b_{2} \neq 0$.

Рассмотрим теперь случай, когда $n \geq 3$. Пусть задан вектор $b^{(n)}=$ $=\left(b_{1}, \ldots, b_{n}\right)$, где $b_{1} \neq 0$, а $b^{(n-1)}=\left(b_{1}, \ldots, b_{n-1}\right)$. Будем использовать обозначения $x^{(k)}=\left(x_{1}, \ldots, x_{k}\right), b^{(k)}=\left(b_{1}, \ldots, b_{k}\right)$,

$$
\varphi^{(k)}\left(x^{(k)}, b^{(k)}\right)=f^{(k)}\left(x^{(k)}\right)+f^{(k)}\left(b^{(k)}+x^{(k)}\right), \quad k=n-1, n .
$$

Предположение индукции состоит в том, что при некотором $n \geq 3$

$$
\left|M\left[\varphi^{(n-1)}\left(x^{(n-1)}, b^{(n-1)}\right)\right]\right| \leq q \quad \forall x^{(n-1)} \in K^{n-1} .
$$

Покажем, что при этом условии

$$
\left|M\left[\varphi^{(n)}\left(x^{(n)}, b^{(n)}\right)\right]\right| \leq q \quad \forall x^{(n)} \in K^{n} .
$$

При заданном $x^{(n)}$ рассмотрим в соотношении

$$
\varphi^{(n)}\left(\tilde{x}^{(n)}, b^{(n)}\right)=\varphi^{(n)}\left(x^{(n)}, b^{(n)}\right),
$$


представляющем собой систему из $C_{n}^{2}$ уравнений над полем $K$ относительно $\tilde{x}^{(n)}=\left(\tilde{x}_{1}, \ldots, \tilde{x}_{k}\right)$, уравнение, отвечающее произведению $x_{1} x_{n}$ (см. (77)):

$$
\tilde{x}_{1} \tilde{x}_{n}+\left(\tilde{x}_{1}+b_{1}\right)\left(\tilde{x}_{n}+b_{n}\right)=x_{1} x_{n}+\left(x_{1}+b_{1}\right)\left(x_{n}+b_{n}\right) \text {. }
$$

Из (83) получаем

$$
b_{1} \tilde{x}_{n}+b_{n} \tilde{x}_{1}=b_{1} x_{n}+b_{n} x_{1}
$$

Согласно условию $b_{1} \neq 0$ отсюда следует, что

$$
\tilde{x}_{n}=b_{1}^{-1}\left(b_{n} \tilde{x}_{1}+b_{1} x_{n}+b_{n} x_{1}\right) .
$$

Значит, при каждом $\tilde{x}^{(n-1)}$, удовлетворяющем соотношению

$$
\varphi^{(n-1)}\left(\tilde{x}^{(n-1)}, b^{(n-1)}\right)=\varphi^{(n-1)}\left(x^{(n-1)}, b^{(n-1)}\right),
$$

имеется не более одного варианта $\tilde{x}^{(n)}=\left(\tilde{x}^{(n-1)}, \tilde{x}_{n}\right)$, удовлетворяющего соотношению (82). Поэтому из (80) следует (81).

Таким образом, неравенства (75) при $b=\left(b_{1}, \ldots, b_{n}\right)$, где $b_{1} \neq 0$, доказаны. Покажем, что перестановка элементов в ненулевом векторе $b=\left(b_{1}, \ldots, b_{n}\right)$ не влияет на результат наших рассуждений.

Возьмем произвольный ненулевой вектор $b^{\prime}=\left(b_{1}^{\prime}, \ldots, b_{n}^{\prime}\right)$ и подстановочную $(n \times n)$-матрицу $G$, которая переводит $b^{\prime}$ в вектор $b$ c $b_{1} \neq 0$ :

$$
G b^{\prime}=b
$$

(здесь и далее все векторы представляются столбцами). Следуя определению (74) отображения $f^{(n)}(x)$, сопоставим матрице $G$ подстановочную $C_{n}^{2} \times C_{n}^{2}$-матрицу $\tilde{G}$, удовлетворяющую условию

$$
\tilde{G} f^{(n)}(x)=f^{(n)}(G x) .
$$

Используя (88) и очевидное равенство $M[\varphi(x)]=M[\tilde{G} \varphi(x)]$ при отображении $\varphi: K^{n} \rightarrow K^{C_{n}^{2}}$, получаем

$$
\begin{gathered}
M\left[f^{(n)}(x)+f^{(n)}\left(b^{\prime}+x\right)\right]=M\left[\tilde{G}\left(f^{(n)}(x)+f^{(n)}\left(b^{\prime}+x\right)\right)\right]= \\
=M\left[\tilde{G} f^{(n)}(x)+\tilde{G} f^{(n)}\left(b^{\prime}+x\right)\right]=M\left[f^{(n)}(G x)+f^{(n)}\left(G b^{\prime}+G x\right)\right] .
\end{gathered}
$$

Отсюда, с учетом (87), находим

$$
\left|M\left[f^{(n)}(x)+f^{(n)}\left(b^{\prime}+x\right)\right]\right|=\left|M\left[f^{(n)}(G x)+f^{(n)}(b+G x)\right]\right| .
$$


Согласно доказанному ранее, $\left|M\left[f^{(n)}(G x)+f^{(n)}(b+G x)\right]\right| \leq q$ для всех $x \in K^{n}$. Лемма 3 доказана.

Пусть $K=G F(2)$ и отображение $f$ удовлетворяет условию (28). Тогда, согласно (75) при любом выборе вектора $x^{1} \neq c^{1}$ уравнение (72) (где $a_{1}=$ $=a_{2}=1$ ) имеет не более 2 решений для $x^{2}$. Следовательно, для числа $N_{1}$ пар $\left(x^{1}, x^{2}\right), x^{1} \neq c^{1}$, удовлетворяющих уравнению (72), выполнено неравенство $N_{1} \leq 2\left(2^{n}-1\right)$. Вместе с тем, при выборе $x^{1}=c^{1}$ число решений уравнения (72) относительно переменной $x^{2}$ равно $N_{2}=2^{n}$, если $c^{2}=f\left(c^{1}\right)$, и равно нулю, если $c^{2} \neq f\left(c^{1}\right)$. Следовательно,

$$
N_{f}=N_{1}+N_{2} \leq 3 \cdot 2^{n}-2 .
$$

Из (69), (73) и (89) получаем (29). Теорема 6 доказана.

Доказательство теоремы 7. Достаточно рассмотреть случай, когда $m=n+C_{n}^{2}$,

$$
f(x)=\left\{f_{1}(x), \ldots, f_{m}(x)\right\}=\left\{x_{i} x_{j}: 1 \leq i \leq j \leq n\right\} .
$$

В уравнении (72), выписанном для отображения (90), произведем замену переменных $y=x^{1}, z=x^{2}$, и запишем векторы $c^{1}$ и $c^{2}$ как $\left(c_{1}, \ldots, c_{n}\right)$ и $\left(c_{i, j}, 1 \leq i \leq j \leq n\right)$ соответственно. Тогда (72) запишется в виде системы уравнений

$$
a_{1} y_{i} y_{j}+a_{2} z_{i} z_{j}+\left(c_{i}-a_{1} y_{i}-a_{2} z_{i}\right)\left(c_{j}-a_{1} y_{j}-a_{2} z_{j}\right)=c_{i, j}, \quad 1 \leq i \leq j \leq n,
$$

относительно неизвестных $y_{1}, \ldots, y_{n}, z_{1}, \ldots, z_{n}$. Раскроем в уравнениях этой системы скобки и разделим ее на подсистемы

$$
\left(a_{2}+a_{2}^{2}\right) z_{i}^{2}-2 a_{2}\left(c_{i}-a_{1} y_{i}\right) z_{i}+F_{i}\left(a_{1} ; y_{i}, c_{i}\right)=c_{i, i}, \quad i=1, \ldots, n,
$$

где $F_{i}\left(a_{1} ; y_{i}, c_{i}\right)=a_{1} y_{i}^{2}+\left(c_{i}-a_{1} y_{i}\right)^{2}$, и

$$
\left[\left(a_{2}+a_{2}^{2}\right) z_{i}-a_{2}\left(c_{i}-a_{1} y_{i}\right)\right] z_{j}-a_{2}\left(c_{j}-a_{1} y_{j}\right) z_{i}+F_{i, j}\left(a_{1} ; y_{i}, y_{j}, c_{i}, c_{j}\right)=c_{i, j},
$$

где $1 \leq i<j \leq n, F_{i, j}\left(a_{1} ; y_{i}, y_{j} c_{i}, c_{j}\right)=a_{1} y_{i} y_{j}+\left(c_{i}-a_{1} y_{i}\right)\left(c_{j}-a_{1} y_{j}\right)$.

Пусть $N_{\left[a_{2} \neq-1\right]}$ - максимальное (по всем $c^{1} \in K^{n}, c^{2} \in K^{n+C_{n}^{2}}$, $\left.a_{1}, a_{2} \in K \backslash\{0\}\right)$ число решений системы (91) для случая, когда $a_{2} \neq-1$. В случае, когда $a_{2}=-1$, для этого числа будем использовать обозначение $N_{\left[a_{2}=-1\right]}$. 
Пусть Char $K=2$. Тогда подсистема (92) принимает вид

$$
\left(a_{2}+a_{2}^{2}\right) z_{i}^{2}+F_{i}\left(a_{1} ; y_{i}, c_{i}\right)=c_{i, i}, \quad i=1, \ldots, n
$$

Если $a_{2} \neq-1$, то при любом значении переменной $y_{i} \in K$ уравнение подсистемы (94) с номером $i=1, \ldots, n$ в случае его разрешимости имеет для переменной $z_{i}$ одно решение (кратное двум). Поэтому

$$
N_{\left[a_{2} \neq-1\right]} \leq q^{n}
$$

Пусть теперь $a_{2}=-1$. В этом случае исходное уравнение (72) после замены переменных $y=x^{1}, z=x^{2}$ принимает вид

$$
a_{1} f(y)+f(z)+f\left(\left(c^{1}+a_{1} y\right)+z\right)=c^{2} .
$$

Согласно лемме 2 (см. (75)) при любом выборе вектора $y \neq a_{1}^{-1} c^{1}$ уравнение (96) имеет не более $q=2^{\alpha}$ решений для вектора $z$. Следовательно, для числа $N_{1}$ пар $(y, z), y \neq a_{1}^{-1} c^{1}$, удовлетворяющих уравнению (96), выполнено неравенство $N_{1} \leq q\left(q^{n}-1\right)$. При $y=a_{1}^{-1} c^{1}$ число решений уравнения (96) относительно вектора $z$ равно $N_{2}=q^{n}$, если $c^{2}=a_{1} f\left(a_{1}^{-1} c^{1}\right)$, и равно нулю в противном случае. Следовательно,

$$
N_{\left[a_{2}=-1\right]}=N_{1}+N_{2} \leq q^{n}(q+1)-q .
$$

Из (95), (97) и (69) получаем (31). Теорема 7 доказана.

Доказательство теоремы 8. Нам опять достаточно рассмотреть случай, когда $m=n+C_{n}^{2}$ и выполнено равенство (90).

Пусть $K=G F\left(p^{\alpha}\right), p \geq 3$. Будем использовать системы уравнений (92), (93). Пусть $a_{2} \neq-1$. При $y \in K^{n}$ и $0 \leq k \leq n-1$ введем обозначения $H_{k}\left(z_{1}, \ldots, z_{k}\right)$ и $h_{k}\left(z_{1}, \ldots, z_{k}\right)$ для множества и числа решений $\left(z_{k+1}, \ldots, z_{n}\right)$ системы, составленной из $n-k$ последних уравнений системы (92) и $C_{n-k}^{2}$ уравнений системы (93) с номерами $(i, j), n-k \leq i<j \leq n$, в которых заданы значения неизвестных $z_{1}, \ldots, z_{k}$ (обозначим ее $(91)_{k}$ ). При $k=0$ аргумент в этом обозначении указывать не будем. Кстати, $H_{0}$ и $h_{0}-$ это множество и число решений системы (91).

Пусть $k=0$. В рассматриваемом случае $a_{2}+a_{2}^{2} \neq 0$ и, согласно уравнениям (92), при любом значении вектора $y$ каждая переменная $z_{i}, i=1, \ldots, n$, имеет не более двух решений.

Если переменная $z_{1}$ принимает одно значение, то

$$
H_{0}=\left\{\left(z_{1}, z^{\prime}\right): z^{\prime} \in H_{1}\left(z_{1}\right)\right\}, \quad h_{0}=h_{1}\left(z_{1}\right)
$$


Если переменная $z_{1}$ имеет два решения, то по крайней мере для одного из них выполнено неравенство (см. уравнение (93) для $i=1, j>1)\left(a_{2}+a_{2}^{2}\right) z_{1}$ $-a_{2}\left(c_{1}^{\prime}-a_{1} y_{1}\right) \neq 0$, и согласно (93), переменные $z_{2}, \ldots, z_{n}$ им определяются однозначно. Другому решению для $z_{1}$ отвечают либо одно определенное, либо все решения системы (91) 1 . Следовательно, $h_{0} \leq 1+h_{1}\left(z_{1}\right)$.

Положим $h_{k}^{*}=\max _{z_{1}, \ldots, z_{k}} h_{k}\left(z_{1}, \ldots, z_{k}\right), k=0, \ldots, n-1$. Из приведенных выше рассуждений следует, что $h_{0}^{*} \leq 1+h_{1}^{*}$.

Аналогичные рассуждения приводят к неравенствам

$$
h_{k}^{*} \leq 1+h_{k+1}^{*}, \quad k=1, \ldots, n-2, \quad h_{n-1}^{*} \leq 2 .
$$

Следовательно,

$$
N_{\left[a_{2} \neq-1\right]} \leq\left|K^{n}\right| h_{0}^{*} \leq q^{n}(n+1) .
$$

Пусть теперь $a_{2}=-1$. В этом случае $a_{2}+a_{2}^{2}=0$, и уравнения (92) и (93) принимают вид

$$
\begin{gathered}
2\left(c_{i}-a_{1} y_{i}\right) z_{i}+F_{i}\left(a_{1} ; y_{i}, c_{i}\right)=c_{i, i} \\
\left(c_{i}-a_{1} y_{i}\right) z_{j}+\left(c_{j}-a_{1} y_{j}\right) z_{i}+F_{i, j}\left(a_{1} ; y_{i}, y_{j}, c_{i}, c_{j}\right)=c_{i, j} .
\end{gathered}
$$

Сопоставим уравнениям (99) и (100) разбиение $K^{n}=U_{1} \cup \ldots \cup U_{n} \cup\left\{y^{0}\right\}$, где $y^{0}=\left(a_{1}^{-1} y_{1}, \ldots, a_{1}^{-1} y_{n}\right)$ и

$$
U_{k}=\left\{y=\left(y_{1}, \ldots, y_{n}\right) \in K^{n}: c_{i}=a_{1} y_{i}, i=1, \ldots, k-1, c_{k} \neq a_{1} y_{k}\right\},
$$

$k=1, \ldots, n$.

Пусть $N_{\left[a_{2}=-1\right]}^{(k)}$ - максимальное число решений системы (91) относительно $z$ при задании неизвестного вектора $y \in U_{k}$, а $N_{\left[a_{2}=-1\right]}^{(*)}-$ число решений $(y, z)$ системы $(91)$, у которых $y=y^{0}$. Тогда

$$
N_{\left[a_{2}=-1\right]} \leq \sum_{k=1}^{n}\left|U_{k}\right| N_{\left[a_{2}=-1\right]}^{(k)}+N_{\left[a_{2}=-1\right]}^{(*)} .
$$

Нетрудно проверить, что (здесь $q=p^{\alpha}$ )

$$
\left|U_{k}\right|=(q-1) q^{n-k}, \quad k=1, \ldots, n
$$

При заданном $y \in U_{k}$ вектор $z=\left(z_{1}, \ldots, z_{n}\right)$, согласно (99) и (100), может принимать не более $q^{k-1}$ значений (при каждом значении $y$ имеется не более $q$ значений для каждой переменной $z_{1}, \ldots, z_{k-1}$ и одно значение для 
каждой переменной $\left.z_{k}, \ldots, z_{n}\right)$. Значит, $N_{\left[a_{2}=-1\right]}^{(k)} \leq q^{k-1}, k=1, \ldots, n$. Очевидно также, что $N_{\left[a_{2}=-1\right]}^{(*)} \leq q^{n}$. Подставив все эти оценки в (101), получим неравенство

$$
N_{\left[a_{2}=-1\right]} \leq n(q-1) q^{n-1}+q^{n} .
$$

Из (98), (102) и (69) получаем (32). Теорема 8 доказана.

Авторы признательны А. М. Зубкову за полезные замечания.

\section{Список литературы}

1. Копытиев В.А., Михайлов В.Г. Теоремы пуассоновского типа для числа специальных решений случайного линейного включения // Дискретная математика. - 2010. - Т. 22. Вып. 2. - С. 3-21.

2. Копытцев В.А., Михайлов В.Г. Теоремы пуассоновского типа для числа решений случайных включений // Математические вопросы криптографии. - 2010. - Т. 1. Вып. 4. - С. 63-84.

3. Копытцев В.А., Михайлов В.Г. О распределении чисел решений случайных включений // Математические вопросы криптографии. - 2011. - Т. 2. Вып. 2. - С. 55-80.

4. Копытиев В.А., Михайлов В.Г. Предельные теоремы пуассоновского типа для обобщенного линейного включения // Дискретная математика. 2012. - Т. 24. Вып. 3. - С. 108-121.

5. Феллер В. Введение в теорию вероятностей и ее приложения. Т. 1. - М.: Мир, 1984.

6. Сачков В.Н. Вероятностные методы в комбинаторном анализе. - М.: Наука, 1978.

7. Михайлов В.Г. Явные оценки в предельных теоремах для сумм индикаторов // Обозрение прикладной и промышленной математики. - 1994. - Т. 1. Вып. 4. - С. 580-617.

8. Фихтенгольи Г.М. Курс дифференциального и интегрального исчисления. - М.: Наука, 1969. 